\title{
Narrativa
}

\section{Quando il Sud e il Nord s'incontrano: nuove pratiche interculturali}

\section{Angela Biancofiore}

\section{(2) OpenEdition}

\section{Journals}

\section{Edizione digitale}

URL: https://journals.openedition.org/narrativa/720

DOI: 10.4000/narrativa.720

ISSN: 2804-1224

\section{Editore}

Presses universitaires de Paris Nanterre

\section{Edizione cartacea}

Data di pubblicazione: 1 décembre 2017

Paginazione: 161-177

ISBN: 978-2-84016-289-6

ISSN: 1166-3243

\section{Notizia bibliografica digitale}

Angela Biancofiore, «Quando il Sud e il Nord s'incontrano: nuove pratiche interculturali», Narrativa [Online], 39 | 2017, online dal 01 décembre 2021, consultato il 14 janvier 2022. URL: http:// journals.openedition.org/narrativa/720 ; DOI: https://doi.org/10.4000/narrativa.720

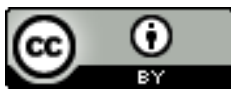

Narrativa est mise à disposition selon les termes de la Licence Creative Commons Attribution 4.0 International. 


\section{Quando il Sud e il Nord s'incontrano: nuove pratiche interculturali}

\section{A Jocelyn Danga e ai giovani poeti del Sud}

I 1 nostro percorso intende esplorare le nuove pratiche dell'interculturalità nell'ambito delle relazioni tra Sud e Nord a partire dal libro di Alessandro Leogrande, La frontiera ${ }^{1}$. Globalmente, possiamo così tracciare le grandi linee del dialogo Nord-Sud alla luce delle recenti trasformazioni geopolitiche e socio-culturali:

1. sviluppo dell'ascolto profondo di un'esperienza;

2. indagine sulle origini dell'alienazione e della deculturazione ;

3. forme della scrittura saggistica e letteraria ;

4. creazione di opere ed eventi, progetti di solidarietà.

Oltre a Leogrande, altri autori oggi in Italia - come Evelina Santangelo, Laila Wadia, Anselmo Botte, Christiana De Caldas Brito - hanno realizzato notevoli esperienze di dialogo interculturale Sud-Nord attraverso la scrittura letteraria e la loro esperienza di vita².

\section{AlCUNE PREMESSE TEORICHE}

Il lavoro critico di alcuni teorici originari del Sud del mondo ci consente di comprendere e analizzare la complessità delle relazioni tra Sud e Nord: in particolare, ci riferiamo a Franco Cassano (Italia), Miguel Benasayag (Argentina),

1. Leogrande, Alessandro, La frontiera, Milano, Feltrinelli, 1995.

2. A partire dalle riflessioni di Camus, di Cassano, di Glissant, di Elytis e di altri autori, abbiamo fondato nel 2011 la rivista web Notos (che in greco significa "Sud"), con il sottotitolo Espaces de la création: arts, écritures, utopies, per dare spazio alle diverse forme di creatività che partono dal Sud, da un Sud non esclusivamente geografico, ma soprattutto simbolico; cfr. l'editoriale http://www.revue-notos.net/?page_id=2. 
Vandana Shiva (India). Il loro peculiare approccio prende in considerazione il fenomeno dell'aggressione economico-culturale esercitata dal Nord: nelle loro opere, emergono alcune questioni fondamentali come la diversità della visione del mondo, la relazione tra progresso e sviluppo tecnologico, la crisi ecologica e la produzione di nuovi valori culturali.

Con Ilpensiero meridiano, Cassano propone un pensiero del Sud, alla ricerca di una ridefinizione dell'idea di progresso e di sviluppo nell'ambito di una società non più fondata sul modello creato e diffuso dal Nord-Ovest del mondo:

Un pensiero del sud, un sud che pensa il sud, vuol dire guadagnare il massimo di autonomia da questa gigantesca mutazione, fissare criteri di giudizio altri rispetto a quelli che oggi tengono il campo, pensare un'altra classe dirigente, un'altra grammatica della povertà e della ricchezza, pensare la dignità di un'altra forma di vita. Significa non pensare il sud o i sud come periferia sperduta e anonima dell'impero, luoghi dove ancora non è successo niente e dove si replica tardi e male ciò che celebra le sue prime altrove $e^{3}$.

Il sociologo italiano rovescia le categorie di una visione ormai inadeguata alla vita del nostro pianeta, in quanto essa non tiene conto della dignità umana $\mathrm{e}$ dell'ecosistema. Il pensiero meridiano è un invito a cambiare radicalmente punto di vista nel segno di un'utopia mediterranea nella quale s'intrecciano antichi e nuovi valori, come l'ospitalità, la convivialità, la lentezza. In particolare, la pratica della lentezza può essere vista come una forma di resistenza alla società dell'efficienza, dove l'essere vivente è strettamente subordinato al suo valore economico.

Parallelamente, il filosofo argentino Miguel Benasayag, molto attivo in Francia e in Italia come pure nel suo paese di origine, l'Argentina, da alcuni anni è impegnato in una ricerca collettiva e individuale che si oppone radicalmente ad una visione dell'umano e della società fondata sulla performance. Gli scienziati del mondo nord-occidentale pretendono di "migliorare" la natura, operando modificazioni genetiche su piante e animali di cui non si conosce ancora l'impatto sulla salute degli esseri viventi.

Una reale comprensione delle modalità di produzione dei valori si rivela centrale per poter far fronte alle trasformazioni della nostra epoca dominata da un'ideologia tecnolatrica. Le domande che il filosofo pone sono di grande attua-

3. Cassano, Franco, Il pensiero meridiano, Bari, Laterza, 1996, nuova edizione 2005, p. 6-7, corsivi miei; dello stesso autore, cfr. Modernizzare stanca. Perdere tempo, guadagnare tempo, Bologna, Il Mulino, 2002. 
lità poiché la tecnologia che si diffonde rapidamente a partire dal Nord-Ovest del mondo impone una sorta di "morale automatica" producendo in noi delle trasformazioni profonde del comportamento di cui non siamo veramente consapevoli. La tecnologia sta contaminando, progressivamente e rapidamente, tutti i modi dell'esistenza; in tal modo, essa introduce il dominio del quantitativo sul qualitativo. Lungi dall'essere tecnofobo, Benasayag è perfettamente cosciente dello sviluppo ultrarapido e irreversibile della tecnologia e della scienza, tuttavia una vera riflessione sul suo impatto nelle nostre vite, in particolare sul modo di comunicare e di comportarsi, è davvero necessaria oggi per comprendere realmente il paradosso contemporaneo:

Nous faisons l'expérience de nouvelles possibilités technologiques qui semblent nous faciliter la vie, mais en même temps, par rapport aux défis cruciaux pour la culture et pour la vie, nous nous sentons de plus en plus impuissants, noyés que nous sommes dans la complexité. Du coup, la nouvelle question qui se pose est la suivante : la puissance technologique actuelle, dans son échange avec le biologique, n'est-elle pas en train de coloniser le monde de la vie en imposant sa logique et sa dynamique? La "déterritorialisation" qu'impose le numérique ne crée-t-elle pas une distance affolante entre l'homme et le monde, entre l'homme et lui-même, par une dislocation du cerveau et du biologique organique en général? ${ }^{4}$

Da alcuni anni un'epistemologa del Sud, Vandana Shiva, originaria dell'India, è diventata una figura emblematica delle lotta (teorica e pratica) contro il modello di sviluppo Nord occidentale e la sua visione del mondo.

È una esponenente di rilievo dell'ecofemminismo, movimento che si oppone ai valori prodotti da una società patriarcale dove la conoscenza tutta femminile del mondo naturale è stata consapevolmente estromessa, in particolar modo nel XVII secolo, durante il fenomeno estremamente violento della caccia alle streghe. Vandana Shiva ha pubblicato il saggio Ecofeminism insieme a Maria Mies, una ricercatrice tedesca: ciò dimostra che è possibile istaurare un dialogo SudNord, Est-Ovest, tra India ed Europa, in nome di valori comuni: la cultura, i diritti delle donne, la protezione della vita sulla terra ${ }^{5}$.

L'ecofemminismo è nato tra gli anni '70 e '80 come protesta delle donne contro i disastri ecologici e contro l'imposizione di un modello dominante

4. Benasayag, Miguel, Cerveau augmenté, homme diminué, Paris, La Découverte, 2016, p. 84; cfr. l'edizione italiana, Il cervello aumentato, l'uomo diminuito, Milano, Erickson, 2016.

5. Cfr. Mies, Maria, Shiva, Vandana, Ecofeminism [1993], trad. fr. Écoféminisme, Paris, L'Harmattan, 1998. 
patriarcale; uno dei primi incontri ispirati a questo nuovo orientamento della ricerca e dell'attività militante è stato Donne e vita sulla terra: convegno sull'ecofemminismo negli anni '80 (Amherst, USA), svoltosi poco dopo il disastro nucleare di Three Miles Island. Le aggressioni dell'industria militare e civile sono state percepite come crimini contro l'ambiente e contro il corpo delle donne, in particolare: in effetti, l'impatto sull'embrione di un disastro chimico come Seveso (1976, Italia) ha toccato molte donne e bambini per varie generazioni. L'ecofemminismo auspica un dialogo profondo tra Sud e Nord del mondo in quanto ridefinisce il concetto stesso di cultura: per i popoli del Sud la cultura è legata ai saperi tradizionali che garantiscono la sopravvivenza (ubicazione delle sorgenti d'acqua potabile, raccolta di erbe mediche, ecc.). Vi è un'enorme differenza nel fatto che per le donne del Nord la cultura è legata all'acquisizione di conoscenze (opere letterarie, cinematografiche, musicali), mentre la cultura della sopravvivenza non è più considerata come un valore in sé:

Les besoins sont habituellement divisés en ce qu'on appelle "les besoins de base" (nourriture, logements, vêtements et autres) et ce qu'on appelle les "besoins supérieurs" comme la liberté la connaissance, etc. La perspective écoféministe, telle qu'elle est exprimée par des femmes activistes, ne reconnait pas ce genre de division. La culture est une part importante de leur lutte pour la subsistance et la vie. Elles identifient la liberté avec leurs liens affectifs et leur travail productif en coopération avec la terre mère, la connaissance est celle de la subsistance essentielle à leur survie. Pour les femmes du Nord opulent ou pour les classes riches du Sud un tel concept d'universalisme et de communautarisme n'est pas facile à saisir. La survie n'est pas considérée comme le but ultime de la vie mais une banalité [...]. C'est précisément la valeur du travail quotidien pour la survie, pour la vie, qui a été érodée au nom des soi-disant valeurs "supérieures".

In realtà, l'attività militante e la ricerca teorica di Vandana Shiva e Maria Mies giungono ad una convergenza poiché si svolgono allo stesso tempo sul piano teorico e pratico; in altri termini, possiamo affermare che nella pratica il sapere non può essere disgiunto dalla lotta per la sopravvivenza che concerne una gran parte dell'umanità. Shiva ci ricorda l'importanza dei valori primari della vita contro i quali è stata dichiarata una guerra lenta, silenziosa ma continua, quella della tecnologia: nei suoi saggi, l'autrice denuncia la diffusione e la vendita da parte delle multinazionali di semi ibridi di numerosissime varietà di piante 
coltivate: ciò ha portato la povertà in molte regioni del Sud del mondo, provocando suicidi in massa di agricoltori'.

Per queste ragioni, la ricercatrice indiana, in numerosi saggi e conferenze, ci invita a "fare la pace con la terra", attirando la nostra attenzione sul carattere sacro della vita:

La pertinence écologique de cette insistance sur la 'spiritualité' repose sur la redécouverte du caractère sacré de la vie, selon lequel la vie sur terre peut seulement être préservée si les gens se mettent à percevoir toutes les formes de vie comme sacrées et les respectent en tant que telles [...] Cette célébration de notre dépendance de la mère terre est bien contraire à l'attitude préconisée par Francis Bacon et ses successeurs, les pères de la science et de la technologie moderne ${ }^{8}$.

\section{Alessandro Leogrande e L’esperienza DELL'INTERCULTURALITÀ: DARE VOCE}

L'incontro di Alessandro Leogrande con i migranti del Sud risale al 1998, quando come volontario dava corsi d'italiano presso un centro sociale sulla Prenestina a Roma9. In tal modo, egli ha potuto incontrare numerosi migranti, ognuno con la sua storia particolare, per comprendere che tutte le singole esperienze di vita partecipano alla Storia ufficiale: Shorsh, faceva parte dei profughi curdi in fuga dagli attacchi del regime di Saddam Hussein; è stato vittima di torture cha hanno lasciato segni indelebili sul suo corpo e per questa ragione è stato dichiarato rifugiato politico. Un giorno egli ha mostrato un video con le immagini del massacro di Halabja al tempo della guerra con l'Iran (1988), dove

7.Cfr.SHIVA, Vandana, The seeds of suicide, https:/ / mmm.commondreams.org/views/2016/05/22/ seeds-suicide\#

8. Mies, Maria, Shiva, Vandana, Écofeminisme, cit., p. 31. Sull'impatto della crisi ecologica nei paesi del Sud, cfr. NixOn, Rob, Slow Violence and the Environmentalism of the Poor, Harvard University Press, 2013.

9. Leogrande è nato a Taranto nel 1977, attualmente vive a Roma. È vicedirettore del mensile Lo straniero e collabora con numerosi giornali e riviste : Il Corriere del Mezzogiorno, Il Riformista, Saturno (inserto culturale de il Fatto Quotidiano). Tra le sue pubblicazioni segnaliamo : Un mare nascosto (L'ancora del Mediterraneo, 2000), Nel paese dei viceré. L'Italia tra pace e guerra (L'ancora del Mediterraneo, 2006), Uomini e caporali. Viaggio tra $i$ nuovi schiavi nelle campagne del Sud (Mondadori, 2008 poi Feltrinelli, 2016), Le male vite. Storie di contrabbando e di multinazionali (Fandango, 2010), Fumo sulla città (Fandango, 2013). Ha curato l'antologia Nel Sud senza bussola. Venti voci per ritrovare l'orientamento (con Goffredo Fofi, L'ancora del Mediterraneo, 2002), e Adriatico (collana digitale Feltrinelli, 2011). Oltre a La frontiera (Feltrinelli, 2015), ha pubblicato Il naufragio. Morte nel Mediterraneo (Feltrinelli, 2011, premi Volponi e Kapuściński), da cui è stata tratta l'opera Katër i Radës. 
ci furono 5000 morti a causa del gas cianuro. Leogrande si accorge di non capire fino in fondo quelle immagini terribili:

Mi accorsi di colpo, che le stavo osservando senza essere in grado di interpretarle. Eppure quelle immagini per Shorsh erano tutto. Non erano un prodotto della storia, erano il suo presente. Non erano una riflessione teorica, erano carne viva ${ }^{10}$.

Così nasce il libro La frontiera, per la volontà di capire quello che c'era prima di arrivare in Italia nell'esistenza di tanti profughi, ovvero "la vita prima del viaggio". Se le motivazioni individuali dei cittadini del Sud del mondo che migrano appaiono comprensibili, le motivazioni storiche lo sono molto meno : "Ad apparirci spesso incomprensibili sono $i$ frammenti di Storia, gli sconquassi sociali, le fratture globali che avvolgono le motivazioni individuali, fino a stritolarle. Incomprensibili perché provengono da un altro mondo" ${ }^{11}$. L'autore cerca di andare al di là della categoria di "vittima» che non spiega niente, scoprendo un Est "molto più a est dei Balcani" e un Sud "molto più a Sud del Maghreb". Portare la nostra attenzione sulla frontiera ci spinge a capire une storia in piena mutazione, in quanto "la frontiera è il termometro del mondo". In effetti, è necessario comprendere da quale mondo fuggono i profughi che preferiscono affrontare viaggi pericolosissimi e anche la morte pur di sfuggire a quel mondo.

In un certo senso, possiamo affermare che la scuola d'italiano costituisce uno spazio di scambio e d'incontro, al di là del puro soddisfacimento dei bisogni primari fornito in una prima fase dai centri di accoglienza sorti in tutta Italia. "Asinitas" è un'altra scuola di italiano per stranieri nella periferia di Roma, a San Paolo fuori le Mura; qui Leogrande incontra Ali, con l'aiuto di Marco, insegnante di lingua. A 21 anni è fuggito dal Darfur perché il suo villaggio e la sua famiglia sono stati violentemente attaccati; quindi è fuggito verso il Ciad fino a Tripoli, dove si è imbarcato su una carretta il cui motore è andato in avaria. Il suo viaggio è stato sconvolgente, ha subito la fame, la sete, l'insolazione, il caldo intenso, ha assistito alla morte di bambini piccoli... un incubo che ancora perseguita il giovane. È stato salvato e curato dagli italiani, poi è diventato in un certo senso un "numero" nelle pratiche umanitarie, un numero tra i salvati da ricordare accanto ai sommersi. Oggi ha 25 anni e fa il venditore ambulante.

Leogrande sente l'urgenza immediata di ascoltare e spiegare la vicenda dei naufraghi e dei migranti; a chi gli chiede perché si è messo ad ascoltare le loro storie, risponde "Perché le frontiere cambiano":

10. Leogrande, Alessandro, La frontiera, cit., p. 12, corsivi miei.

11. Ibid., p. 14. 
Le frontiere cambiano non rimangono mai fisse. Si allarga l'Europa e cambiano i punti di ingresso. Scoppiano guerre, cadono dittature, esplodono intere aree del mondo e si aprono nuovi varchi. I varchi a loro volta creano un mondo, una particolare società di confine che definisce le sue regole e i suoi ruoli all'interno. Sono a tutti gli effetti dei porti franchi. Ma poi anche questi mutano nel tempo, e vengono sostituiti da altri porti franchi ${ }^{12}$.

L'autore ha saputo offrire un ascolto profondo che gli ha permesso di comprendere - e far comprendere - le mutazioni antropologiche, economiche, geopolitiche per rompere quell'insopportabile silenzio intorno ai naufragi prodotto da una pericolosa diffusione dell'indifferenza.

Leogrande racconta la storia di un altro migrante, Hamid: di origine somala, è sopravvissuto a uno dei più grandi naufragi davanti alle coste della Libia il 6 maggio 2011. In realtà, il capitano aveva consapevolmente provocato il naufragio presso le coste poiché sapeva che non ce l'avrebbero fatta ad attraversare il mare con quell'imbarcazione. Sapendo nuotare, il giovane è riuscito a salvarsi e a salvare altri naufraghi, anche se su 750 persone, ci sono stati 650 morti.

Grazie alla testimonianza di un altro cittadino eritreo in Italia, Gabriel Tzeggai, Leogrande arriva a ricostruire la catastrofica situazione del paese africano che è all'origine della migrazione in massa: dopo la conquista dell'indipendenza nel 1993, scoppia un nuovo conflitto con l'Etiopia nel 1998, il regime eritreo si trasforma in dittatura e proclama il servizio di leva obbligatorio, di conseguenza oggi le carceri e i centri di detenzione sono pieni di cittadini che si oppongono al regime; la storia dell'Eritrea suggerisce all'autore un curioso parallelo con le vicende di un'altra ex-colonia italiana, l'Albania.

In realtà, ogni mese un numero importante di Eritrei fugge da una situazione equivalente a uno stato perenne di guerra che dura dal 1940 a oggi: oltre all'Eritrea, si osserva oggi un flusso migratorio proveniente da altri paesi africani come l'Uganda, la Tanzania, l'Etiopia.

Con La frontiera, Alessandro Leogrande ha reso comprensibili e ha dato un volto umano alle vite dei migranti e alla loro Storia: in realtà, per arrivare a istaurare un ascolto profondo è necessario operare una trasformazione su noi stessi per disporci a una ricettività totale. E qui la narrazione acquista un ruolo fondamentale in quanto si ricollega alla grande tradizione della letteratura orale:

Bisogna farsi viaggiatori per decifrare i motivi che hanno spinto tanti a partire e tanti altri ad andare incontro alla morte. Sedersi per terra intorno ad un

12. Ibid., p. 25 . 
fuoco e ascoltare le storie di chi ha voglia di raccontarle, come hanno fatto altri viaggiatori fin dalla notte dei tempi ${ }^{13}$.

\section{Un nuovo sguardo sul Sud: il caso dell'Africa}

In tempi recenti, il continente africano ha vissuto eventi che hanno profondamente marcato la sua Storia producendo mutazioni profonde: tra l'altro, la fine dell'apartheid nel Sudafrica (1991), la guerra civile in Rwanda (aprile-luglio 1994, 800000 vittime in gran parte Tutsi), le primavere arabe nel 2011. Si tratta di importanti segnali di una vasta trasformazione in atto: la guerra civile in Rwanda ha fatto prendere conscienza che non tutti i mali dell'Africa provengono dall'uomo bianco, è stata la fine del mito di un'Africa "felice" prima della colonizzazione. Le primavere arabe, indipendentemente dal loro impatto sugli attuali apparati di governo nei paesi nordafricani, hanno rivelato una profonda volontà di cambiare, una richiesta pressante di democrazia e di trasparenza. Inoltre possiamo osservare che, malgrado la grande diversità culturale, il popolo africano resta profondamente legato a grandi figure carismatiche come Nelson Mandela (Afrique du Sud), Thomas Sankara (Burkina Faso), Patrice Lumumba (RDC Congo).

Progressivamente, nuove pratiche si fanno strada anche nell'ambito degli studi antropologici, come l'“antropologia reciproca" che intende sviluppare la decolonizzazione dello sguardo di noi occidentali sul Sud del mondo. Uno dei progetti che ha adottato questo nuovo approccio è stato Transcultura: tra il 1988 e il 2011 alcuni ricercatori originari dell'Africa e dell'Asia hanno condotto indagini etnologiche in Francia e in Europa che sono state poi pubblicate in due volumi: Sguardi venuti da lontano, e Le renversement du ciel ${ }^{4}$.

Possiamo citare, tra gli altri testi, il racconto di un griot, il cantastorie africano Diawne Diamanka, che descrive la città di Bologna dal suo particolare punto di vista culturale, rovesciando completamente l'immagine che abbiamo di noi stessi e delle nostre forme di vita nord-occidentali.

Nella prefazione al volume possiamo osservare una precisa volontà di scrollarsi di dosso lo sguardo colonizzatore del Nord del mondo, in opposizione ad un'Europa che ha conservato per secoli il monopolio dello sguardo e dell'interpretazione.

13. Ibid., p. 313.

14. Cfr. Le Pichon, Alain, Caronia Letizia (a cura di), Sguardi venuti da lontano. Un'indagine di Transcultura, Milano, Bompiani, 1991; Le Pichon, Alain, Sow, Moussa (a cura di), Le renversement du ciel, con prefazione e testi di Umberto Eco, Paris, CNRS, 2011. 
Uno sguardo lontano, troppo lontano dalla vita, rischia di fermarsi alla pura apparenza dei fenomeni, creando una serie di stereotipi difficili da eliminare.

La presenza dei trofei della colonizzazione nei musei del Nord non fa altro che confermare il lungo processo di divorazione del mondo "Sud" da parte del mondo "Nord", divorazione di beni, risorse, culture (basti pensare alle polemiche sorte intorno al Musée de l'Homme e al più recente Musée des arts premiers di Parigi).

Secondo Umberto Eco, che ha sostenuto e animato il progetto Transcultura fondato sull'antropologia reciproca, comprendersi tra culture diverse non significa valutare quanto costa a ognuno per arrivare a essere uguali, ma "comprendere reciprocamente ciò che ci separa e accettare questa diversità"15. Etnologi, filosofi, artisti e scrittori lavorano attualmente a progetti che mirano a decentralizzare il pensiero e superare una visione monopolizzata dal Nord-Ovest del mondo, "fier de lui-même et de ses lumières", convinto della sua missione civilizzatrice. Nell'antologia letteraria a cura di Alain Mabankou e Michel Le Bris, L'Afrique qui vient ${ }^{16}$, scopriamo una nuova letteratura africana non più intenta a designare l'uomo bianco colonizzatore come unico responsabile del degrado, e che oggi si sviluppa grazie all'emersione di nuovi mondi culturali.

In effetti, assistiamo allo sviluppo di nuove forme di cultura nelle metropoli, vere "centrali creative" (Kinshasa, Lagos, Johannesburg), grazie all'attività di musicisti, cineasti, scrittori, artisti nati dal "caos-mondo" e che, con la loro vitalità, sono in grado di trasformarlo. In particolare, possiamo notare lo sviluppo di nuove forme artistiche legate alla cultura urbana: slam, hip hop, rap ${ }^{17}$.

In sostanza, il punto di partenza per l'istaurazione di un vero dialogo SudNord risiede nel processo di decolonizzazione del pensiero: a questo proposito, il filosofo Achille Mbembé mette in guardia contro la pretesa occidentale di "ricapitolare il linguaggio", di imporre un'interpretazione delle forme nelle quali l'evento umano può emergere fino ad esercitare un monopolio sull'idea stessa di futuro ${ }^{18}$.

Nell'ambito delle relazioni Nord-Sud, ciò che può sembrare normale e ordinario non lo è affatto dal punto di vista di un altro universo culturale: l'esempio ci viene dato da Aminata Traoré, figura di spicco del panorama africano, ex-Ministro

15. Eco, Umberto, Prefazione, in Le Pichon, Alain, Caronia, Letizia (a cura di), Sguardi venuti da lontano. Un'indagine di Transcultura, cit., p. 12.

16. Le Bris, Michel, Mabanckou, Alain (a cura di), L'Afrique qui vient, Paris, Hoëbeke, 2013.

17. Ibid., p. 9.

18. Cfr. Mbembé, Achille, Sortir de la grande nuit, Paris, La Découverte, 2011, p. 10, vedi anche KABOU, Axelle, Et si l'A frique refusait le développement?, Paris, L'Harmattan, 2000. 
della cultura et del Turismo del Mali. In un articolo del 2006, ha dichiarato che, paradossalmente, le opere d'arte africane hanno diritto di cittadinanza laddove gli africani non hanno diritto al soggiorno. Per questa ragione, ha deciso di dare le dimissioni dal comitato del Musée des Arts premiers (Parigi):

Le Musée du Quai Branly est bâti, de mon point de vue, sur un profond et douloureux paradoxe à partir du moment où la quasi-totalité des Africains, des Amérindiens, des Aborigènes d'Australie, dont le talent et la créativité sont célébrés, n'en franchiront jamais le seuil compte tenu de la loi sur l'immigration choisie $^{19}$.

La scelta coraggiosa di Aminata Traoré, derivante da una profonda riflessione sulle forme di patrimonio culturale dell'umanità, ci spinge a intraprendere quel processo di decolonizzazione del pensiero senza il quale non è possibile fondare un vero dialogo interculturale.

\section{IL DIALOGO NORD-Sud NELLE PRATICHE INTERCULTURALI}

È difficile costruire un dialogo fecondo tra Sud e Nord fondato unicamente sulla denuncia, sulla collera, sullo sdegno. Ciò che invece appare indispensabile è lavorare insieme nel quadro di un progetto comune, federatore, fondato sulla non violenza e, più generale, sul rispetto del pianeta. Particolarmente significativa a questo proposito è l'esperienza di Sebastião Salgado, fotografo originario del Brasile, narrata nel film documentario di Wim Wenders e Juliano Ribeiro Salgado, Il sale della terra (2014).

La denuncia non basta, bisogna poter ricostruire insieme: Salgado ha fotografato per anni le vittime delle guerre nella speranza di poter far nascere un mondo migliore, e invece la violenza persiste, anzi si intensifica. Con il massacro nel Rwanda, il fotografo giunge a un punto di non ritorno, non vuole più scattare fotografie, abbandona il mestiere di foto reporter perché la violenza sulle popolazioni ha superato ogni limite del rappresentabile.

Proprio in quel momento di crisi, sua moglie gli propone di restaurare l'antica foresta pluviale in Brasile, nella regione di Minas Gerais, laddove gli uomini hanno distrutto gli alberi desertificando la terra (qui risiedeva in effetti il padre del fotografo che ha contribuito a questa distruzione).

19. Traoré, Aminata, "Ainsi nos œuvres d'art ont droit de cité là où nous sommes, dans l'ensemble, interdits de séjour", in RESF, 27 giugno 2006, http:/ / www.educationsansfrontieres.org/article.php3?id_article $=568$. 
L'operazione di riforestazione è stata organizzata e realizzata con successo, le piantine si sono sviluppate rapidamente e gli antichi alberi della foresta hanno riconquistato il territorio perduto: nasce così l'Istituto terra che è stato dichiarato "parco protetto" del Brasile; Salgado ha ripreso a fotografare, questa volta non più focalizzandosi sulle vittime della guerra o dello sfruttamento, ma sulla capacità di rigenerazione della terra.

L'esperienza esistenziale e professionale di Salgado ci permette di capire un aspetto centrale: accanto all'attività di denuncia, abbiamo innanzitutto bisogno della linfa vitale che alimenta il dialogo tra umano e natura, tra esseri umani di culture diverse.

Nell'ambito delle pratiche interculturali che si distinguono oggi in Italia per il loro interesse, possiamo citare il progetto dell'università di Palermo, svolto in collaborazione con il Comune della Città: I Saperi per l'inclusione. All'interno del progetto, rivolto a 100 donne immigrate di Palermo (tra cui 50 ex-vittime della tratta), Martino Lo Cascio ha intervistato le donne che partecipano ai corsi di italiano e al laboratorio di taglio e cucito, per invitarle a cantare le ninne nanne dei loro paesi d'origine. Le canzoni raccolte saranno inserite nel film del regista, Not $(t)$ e d'oro, che servirà a raccogliere fondi per aiutare le donne e i bambini vittime di violenza in Madagascar.

Il bellissimo video intitolato Everyday capisci niente - Donne immigrate intonano ninne nanne da tutto il mondo (regia di Martino Locascio e Antonio Macaluso) illustra bene questa occasione di scambio tra Sud e Nord, in quanto il patrimonio orale delle donne migranti viene in tal modo valorizzato e inserito nel tessuto sociale : il canto delle donne, tutto il loro mondo affettivo e arcaico si tramanda attraverso le ninna nanne. Le parole, i gesti, la voce, gli sguardi delle donne del Sud esprimono in primo luogo la cura (care) che è una prerogativa dell'universo femminile poiché le donne, per tradizione e da tempi remoti, "curano", ovvero si occupano degli altri, delle persone più fragili (bambini, anziani, malati) ${ }^{20}$.

I progetti di inclusione proposti da ItaStra di Palermo promuovono attivamente la diversità culturale, l'alfabetizzazione e la salvaguardia del patrimonio orale delle donne migranti. Se pensiamo che queste donne africane sono anche state vittime della tratta, il progetto di inclusione e alfabetizzazione acquista un alto valore, poiché esprime la volontà di riabilitare il dialogo sociale con soggetti fragili, che restano ai margini della società italiana. La scuola

20. Il video è visibile su youtube "Everyday capisci niente. Ricordare ninne nanne". Apprendere l'italiano. Immagini da un laboratorio, https://www.youtube.com/ watch? $\mathrm{v}=\mathrm{mELN} 5 \mathrm{ho} 0 \mathrm{Ajw}$ 
d'italiano ha organizzato inoltre un laboratorio di poesia attraverso il quale le donne africane hanno potuto esprimere frammenti delle loro storie ${ }^{21}$.

Tra le pratiche interculturali in grado di avvicinare il mondo Sud e il mondo Nord vi è anche il teatro che si può trasformare in un vero strumento di dialogo interculturale: ItaStra e Teatro Libero di Palermo hanno creato insieme il progetto Il teatro delle differenze: l'arte crea in tal modo un'esperienza di confronto tra mondi e culture diverse attraverso il laboratorio che si è svolto con i formatori del Teatro Libero Palermo (maggio-giugno 2017). Il progetto globale, intitolato La forza della lingua. Percorsi di inclusione per soggetti fragili, consente agli studenti che apprendono l'italiano presso la Scuola di Lingua Italiana per Stranieri (ItaStra) dell'Università degli Studi di Palermo di acquisire le tecniche teatrali di base in modo da conoscere meglio se stessi e costruire un rapporto più consapevole con l'Altro e con l'Ambiente.

La pratica della narrazione assume un ruolo centrale in numerose iniziative contro l'esclusione al fine di incentivare e far crescere il dialogo tra Nord e Sud. A questo proposito, possiamo menzionare le attività della Fondazione Lettera27 che ha concepito il progetto Luoghi Comuni - piccole storie migranti: attraverso le parole di italiani e migranti, giovani e adulti, il progetto documenta la produzione di senso e di cultura che deriva dal confronto tra modelli, saperi, e stili di vita diversi.

La città di Palermo ha collaborato al progetto, attraverso il CISS-Cooperazione Internazionale Sud Sud, nell'ambito di Talenti integrati, un progetto nato nel 2012 e cofinanziato dall'Unione Europea (Fondo Europeo per l'Integrazione dei cittadini di paesi terzi) e dal Ministero dell'Interno (Dipartimento per le Libertà Civili e l'Immigrazione) $)^{22}$.

$\mathrm{Da}$ alcuni anni a Bologna l'associazione culturale Annassim, contribuisce all'avvicinamento di diversi mondi culturali creando numerose occasioni di dialogo tra donne del Nord e del Sud: la sua azione è volta principalmente alla valorizzazione del patrimonio culturale delle donne migranti del Sud e dell'Est, in particolare tramite l'insegnamento delle loro lingue e alfabeti nelle scuole italiane; l'associazione favorisce essenzialmente le pratiche collettive, ha istituito corsi di tessitura, diversi laboratori sull'uso dell'henné, sull'arte della ceramica,

21. https://www.youtube.com/watch?v $=m k U o 1 \mathrm{cmaQEo}$. Vedi anche i progetti didattici di ItaStra sull'alfabetizzazione alla Mostra del libro didattico di Milano http:// www.unipa.it/strutture/scuolaitalianastranieri/.

22. Per maggiori informazioni si veda il sito http://www.lettera27.org/index.php?id.... 
il maquillage, la poesia, ha creato orti urbani per migliorare le relazioni nei quartieri e con l'ambiente ${ }^{23}$.

Anche in Francia si sente l'urgenza di intensificare le pratiche interculturali grazie all'attività di numerose associazioni e istituzioni. Possiamo citare in proposito un'azione recente: il Rectorat di Montpellier ha promosso la mostra fotografica di una studentessa, Lisa Barthélémy, nell'ambito del progetto Alter-égaux (colloque Florilège, maggio 2017, promosso dalla "missione letteratura" a cura dei professori di lettere Frédéric Miquel e Marie Gola).

Lisa Barthélémy, studentessa presso l'università Paul-Valéry di Montpellier, ha potuto realizzare una particolare esperienza del dialogo interculturale tra Nord e Sud attraverso il volontariato in un campo di rifugiati minorenni gestito dalla Croce Rossa a Barvaux, in Belgio. Più precisamente, ha partecipato, insieme ad altri studenti di tutta l'Europa, a un cantiere collettivo volto a migliorare le condizioni di vita dei ragazzi residenti nel campo. In seguito, Lisa ha sentito il bisogno urgente di testimoniare, con la fotografia, la vita dei ragazzi che ha incontrato, giovani come lei che vogliono avere l'opportunità di studiare, una possibilità che viene loro negata nei paesi di origine. Ne è nata una mostra fotografica itinerante, La patience des papillons, che ha già suscitato numerose occasioni di dibattito in tutti i luoghi in cui è stata accolta. Nel suo blog, Lisa dice di aver imparato molto dai ragazzi rifugiati, ha imparato la pazienza, il coraggio, "un coraggio che si può appena immaginare"24.

23. Cfr. Di Marco, Lella, Lotuso, Paola (a cura di), I saperi delle donne. Il patrimonio culturale delle donne migranti nella cura della persona e la gestione del quotidiano, Bologna, Martina editore, 2008. Vedi anche il sito http://saperidelledonne.blogspot.fr/.

24. Così Lisa esprime la sua esperienza nel campo profughi: “Il n’y avait pas de différence entre nous. La langue bien sûr, certains reflexes. Mais sinon, pas de différence. Nous avons mis du temps à nous apprivoiser les uns les autres. Pourtant au bout de deux semaines, j'avais rencontré des personnes incroyables, j'avais construit des amitiés fortes, impérissables. J'ai tellement appris avec eux. J'ai appris la peur de demain, la véritable solitude, l'implacable séparation, l'horreur de l'attente, la haine de l'injustice. J'ai appris malgré tout ça, à rire quand même, à rester léger, j'ai appris les amitiés d'un jour, la force d'un regard, le prix d'un sourire. J'ai appris le courage. Vraiment. Un courage que l'on n'imagine à peine. [...] Par cette exposition, je veux mettre des visages sur cette masse de personnes, mettre des identités, des portraits de vie, pour individualiser cette situation de crise, pour rendre les enjeux réels et concerner les gens" (Barthélémy, Lisa, La Patience des papillons, https://fr.ulule.com/ patience-des-papillons/). 


\section{EdUCARE AL DIALOGo SUd-Nord}

Tutte le pratiche interculturali di cui abbiamo parlato hanno un comune obiettivo: educare al dialogo Sud-Nord, avvicinare mondi culturalmente distanti, insegnare a vivere insieme.

L'educazione al dialogo interculturale, tramite azioni in cui sono coinvolti adulti, giovani e bambini può contribuire pienamente alla costruzione di una società in cui tutti i mondi possano trovare il loro spazio vitale, federati intorno a progetti valorizzanti.

È quindi essenziale individuare le grandi linee di un'azione culturale educativa che mira alla comprensione delle differenze; di fronte alle azioni terroristiche degli ultimi anni che hanno principalmente colpito Inghilterra, Francia, Belgio e Germania, è davvero urgente incentivare azioni e progetti collettivi che consentano di ristabilire il dialogo Sud-Nord, Est-Ovest, al di là degli stereotipi religiosi, sociali, culturali.

Innanzitutto è necessario partire proprio dalla nostra mente, spostando il cosiddetto "centro del mondo", non più situato a Nord-Ovest, per poter liberare la mente dagli stereotipi e dall'avversione per tutto ciò che è diverso da noi. È indispensabile creare aperture, far comprendere i rapporti di interdipendenza tra Sud e Nord, in quanto i due mondi non potrebbero sussistere l'uno senza l'altro. È giunto il tempo di "fare la pace con la terra", per avviare pratiche di ecologia ambientale e sociale. Possiamo ormai allargare l'orizzonte semantico dell'ecologia par poter pensare un'ecologia della mente che non possiamo disgiungere dall'ecologia sociale (che riguarda la società, come ad esempio i fenomeni migratori), e dall'ecologia ambientale: pertanto, appare indispensabile lo sviluppo dell'ecocritica, ovvero di una critica delle opere letterarie (e artistiche in genere) in grado di far comprendere le relazioni di interdipendenza tra natura e cultura, tra umano e non umano, tra i mondi culturali ${ }^{25}$.

25. Sul rapporto tra ecologia e filosofia, cfr. Guattari, Felix, Les Trois écologies, Paris, Galilée, 1989; Iovino, Serenella e Oppermann, Serpil (eds), Material Ecocriticism, Bloomington and Indianapolis, Indiana University Press, 2014; CALlicotT, Baird John, Ethique de la terre, Marseille, Wildproject, 2010; Alaimo, Stacy, Bodily Natures. Science, Environnement and Material Self, Bloomington, Indiana University press, 2010; LARRÈRE, Catherine, Du bon usage de la nature, Paris, Aubier, 1997, Flammarion, 2009. Sull'ecocritica, cfr. Iovino, Senella, Ecologia letteraria Una strategia di sopravvivenza, Milano, Edizioni Ambiente, 2006; Glotfelty, Cheryll \& Fromm, Harold, The Ecocriticism Reader: Landmarks in Literary Ecology, University of Georgia Press, 1996; Biancofiore, Angela, "Ethique de la terre et appropriation du vivant", in Notos, n. 3/2015, http://www. revue-notos.net/?page_id=808. 
L'ecocritica, senza diminuire il contributo dell'analisi delle forme artistiche, si concentra sul ruolo della letteratura oggi nell'ambito di una strategia di sopravvivenza.

L'azione educativa tocca in particolar modo le scuole, le università, i luoghi dove si svolgono pratiche collettive (teatri, biblioteche, centri culturali) incentivando quindi le relazioni con i centri decisionali a livello locale (Comuni, Provincie, Regioni), nazionale o internazionale (ministeri, organismi internazionali). La relazione di cura (care) che si stabilisce con i cittadini del Sud che vivono nel Nord del mondo ci obbliga a uscire dall'indifferenza: a livello filosofico e nelle pratiche, è oggi urgente sviluppare una visione fondata sul ruolo centrale del care nella sfera del dialogo sociale e interculturale ${ }^{26}$.

In realtà, durante il suo lavoro di inchiesta poi confluito nel volume La frontiera, Alessandro Leogrande ha fatto l'esperienza del care: di fatto si è "preso cura" delle storie dei naufraghi, e le ha raccontate dando loro voce; vi è il caso di altri scrittori, come Anselmo Botte che ha preso cura della storia dei migranti e le ha raccontate poi alla prima persona nel volume Mannaggia la miseria ${ }^{27}$; possiamo inoltre citare l'esperienza di Evelina Santangelo che si è "presa cura" delle storie dei migranti e dei giovani siciliani nel suo romanzo Senzaterra ${ }^{28}$, e il caso ha voluto che la scrittrice abbia pure assistito direttamente a uno sbarco di migranti sulle coste della Sicilia. D'altra parte, Laila Wadia, scrittrice italo-indiana, ha saputo prendersi cura delle storie delle donne straniere provenienti da diversi mondi culturali, riunite in un condominio precario nella città di Trieste...

Possiamo evocare numerosi altri scrittori come Christiana de Caldas Brito, scrittrice italo-brasiliana, o Carmine Abate, scrittore italiano e arbëresh... Tutti questi autori che scrivono in lingua italiana si trovano oggi riuniti in un'antologia pubblicata nel 2016 e significativamente intitolata Soyons le changement... in riferimento alla nota frase di Ghandi: "Sii il cambiamento che vuoi vedere nel mondo" 29 . Per poter innescare la trasformazione, il punto da cui si parte è

26. Sulla nozione di care (o cura) facciamo riferimento ai lavori di GiLLIGAN, Carol, Une voix différente. Pour une éthique du care, Paris, Champs-Flammarion, 2008; Laugier, Sandra, Molinier, Pascale, Paperman, Patricia, Qu'est-ce que le care?: Souci des autres, sensibilité, responsabilité, Paris, Payot, 2009.

27. Вотте, Anselmo, Mannaggia la miseria, Roma, Ediesse, 2009.

28. Santangelo, Evelina, Senzaterra, Torino, Einaudi, 2008.

29. Summa, Romano, Ben Abdallah, Sondes (a cura di), Soyons le changement... Nouvelles tendances dans la littérature italienne contemporaine, prefazione di Angela Biancofiore, trad. fr. Manon Rentz, Romano Summa, Sondes Ben Abdallah, Euromédia \& Levant, 2016. 
proprio la nostra mente: solo in tal modo l'onda di solidarietà potrà estendersi a tutta la sfera sociale e politica.

In conclusione, è lo spazio del narrare che consente di riunire teoria e pratica del dialogo Nord-Sud: raccontare storie, ascoltare, trasmettere al fine di ricostituire la relazione che unisce, per rafforzare i legami di solidarietà, per non cedere alla minaccia di un preteso scontro di civiltà; per infine scoprire che ciò che ci unisce è molto più importante ed essenziale di ciò che ci separa.

Possiamo quindi chiudere il nostro itinerario dedicato alle relazioni interculturali Sud-Nord, facendo dialogare (a distanza) due poeti, nati nelle terre del Sud del mondo: Italia del Sud e Africa. Si tratta di Comasia Aquaro (Martina Franca, Taranto, Italia) e Jocelyn Danga (Kinshasa, Repubblica del Congo):

\section{La luce che non muore, Comasia Aquaro}

La vita è stretta

troppo perché ci passi

un angelo.

E allargare l'aria

non è possibile.

Tanto vale stringerci

come in un unico

amore di madre

che scorre da essere a essere ${ }^{30}$.

Un seul amour, Jocelyn Danga ${ }^{31}$

Un seul soleil pour sept milliards d'êtres

Un seul tableau pour sept milliards de peintres

Sept milliards de bâtons pour une seule flamme

Un seul paradis pour sept milliards d'âmes

Pour sept milliards de rois, un seul sceptre

Pour sept milliards de messagers, une seule lettre

Sept milliards d'étoiles pour un seul ciel

Sept milliards de couleurs pour un seul arc-en-ciel

30. Aquaro, Comasia, La luce che non muore, con testo francese a fronte, trad. Pascale Climent, prefazione di Angela Biancofiore, Montpellier, Levant, 2017.

31. Con questa poesia il giovane poeta africano (oggi ventenne) ha vinto il primo premio del concorso internazionale di poesia "Matiah Eckhard" 2016. Ha anche ottenuto lo stesso anno il premio "Léopold Senghor". 
Pour un seul jardin, sept milliards de roses

Pour une seule histoire, sept milliards de proses

Sept milliards de voix pour un seul chœur Sept milliards de pétales pour une seule fleur Sept milliards de mots pour une seule note

Pour une seule chanson, sept milliards de notes

Un seul silence pour sept milliards de bouches

Un seul rêve pour sept milliards de couches

Un même espoir pour sept milliards de cœurs

Un seul sourire pour sept milliards de bonheurs

O doux iris ! Il n'en sera jamais de trop

Pour chacun, sept milliards d'un seul parcours

Pour tous, sept milliards d'un seul amour

Sept milliards de doigts dans un seul anneau.

Angela BiAnCofiore

Université Paul-Valéry-Montpellier 3

(Centre de Recherche LASI - Equipe d'accueil LLACS) 
